\title{
The effect of adapting and test field size upon threshold during early dark adaptation
}

\author{
FREDERICK L. KITTERLE and LAWRENCE E. LEGUIRE \\ University of Toledo, Toledo, Ohio 43606
}

\begin{abstract}
The course of early dark adaptation appears to be differentially influenced by adapting field size. In general, longer time courses were found when adapting and test fields were similar in size. Comparisons among configurations in which adapting and test fields were similar in relative size but different in absolute size indicated a longer time course for the smallest configuration.
\end{abstract}

Kitterle and Leguire (1975) showed that adapting field size influences the magnitude of the threshold drop during the course of early dark adaptation. Specifically, the threshold drop was greatest when both test and adapting fields were similar in size and was smaller when the adapting field was larger than the test field. If incremental thresholds are measured upon adapting fields of varying diameters (Frumkes \& Kraft, 1972; Frumkes \& Sturr, 1968; Markoff \& Sturr, 1971; Ratoosh \& Graham, 1951), backgrounds similar in size to the test field raise thresholds more than do those that are larger in size.

Kitterle and Leguire (1975) hypothesized that the relationship between the magnitude of the threshold elevation before the offset of the adapting field and the threshold drop after the adapting field is turned off may be due, in part, to a similar mechanism, namely, lateral border interference and its decay. In the case in which the test field is superimposed upon the background, the contours of the adapting field, being close to those of the test field, inhibit the contours of the test field. The magnitude of this border interference progressively diminishes as the size of the adapting field is increased relative to that of the test field. Consequently, for similar-sized test and adapting fields, incremental thresholds would be more elevated than test and adapting fields of different sizes. The time course of early dark adaptation should be longer if early dark adaptation reflects a release from the inhibitory influences established at the time the adapting field was visible. The present experiment tests this hypothesis.

\section{METHOD}

\section{Subjects}

There were two observers in this experiment, L.E.L. (23 years), who was experienced in making these kinds of judgments, and J.M. (22 years), who was naive about the hypotheses of this experiment.

\footnotetext{
Apparatus

The apparatus used to present the stimuli was a three-channel Maxwellian view optical system. A detailed description of this
}

system and its calibration is given in an earlier publication (Kitterle \& Leguire, 1975).

Two red fixation points ( $2 \mathrm{~min}$ in diameter) were continuously visible above and below the position in which the test field was presented. Observers fixated between the two points.

\section{Procedure}

The observer preadapted to a 3.4- $\log -\mathrm{mL}$ adapting field for $7 \mathrm{~min}$ at the beginning of each experimental session. After preadaptation, the test flash followed at some preset interstimulus interval (ISI) either before (negative ISI) or after (positive ISI) the adapting-field offset.

The method of adjustment was used to set the test flash to threshold. Eight threshold determinations were made for each condition.

Two seconds of darkness followed the test flash before the adapting field came on again. The adapting field remained on for $15 \mathrm{sec}$ before the cycle was repeated. The test flash duration was $20 \mathrm{msec}$. Viewing was monocular, with the stimuli presented to the left eye. No artificial pupil was used.

\section{RESULTS}

The results of this experiment are presented in Figure 1A for L.E.L. and Figure 1B for J.M. In Figure 1, early dark adaptation was determined with a variety of adapting field sizes. The test field size was constant for this set of measurements; it was $15.8 \mathrm{~min}$. There are two aspects of Figure 1 that should be noted.

The first aspect concerns the negative ISIs, when incremental thresholds are in effect being determined, since the test field is then presented upon the adapting field. Notice that increases in the size of the adapting field result in a progressive decrease in the magnitude of the incremental threshold.

The second aspect of Figure 1 is the threshold drop measured from $0 \mathrm{msec}$ to $250 \mathrm{msec}$. In general, the drop appears to be greatest when both test and adapting fields are similar in size. It appears that no further decrease in the magnitude of the threshold drop occurs beyond an adapting size of $31.6 \mathrm{~min}$. For incremental thresholds, no further decreases in threshold drop were found beyond this adapting field size.

Additional measurements were made by L.E.L. for test fields of $31.6 \mathrm{~min}$ and $56.1 \mathrm{~min}$ and a range of 

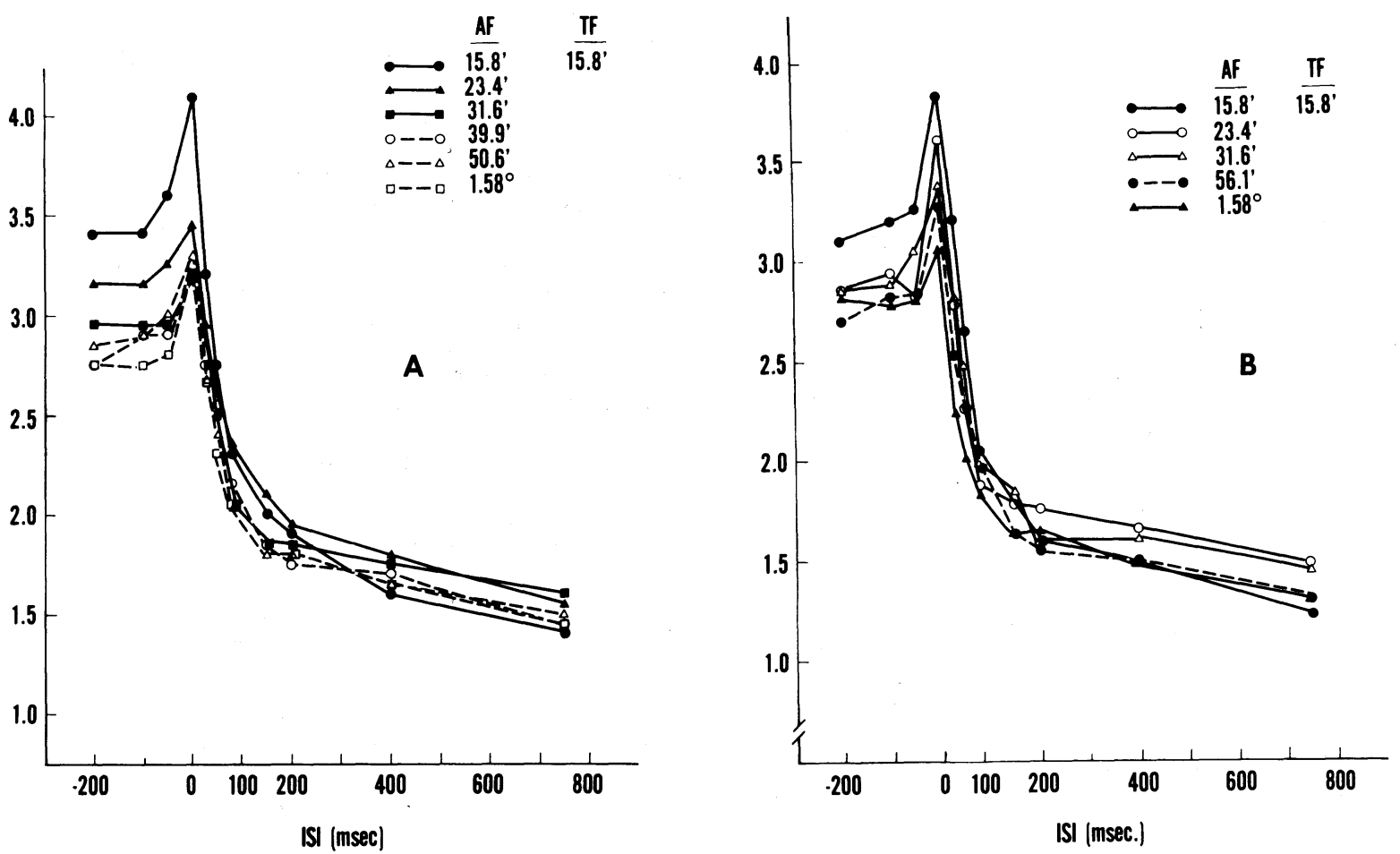

Figure 1. Log threshold elevation as a function of ISI for a 15.8-min test spot. Each curve is for a different adapting size. Standard deviations equal the size of the plotted points for all curves shown in all figures. (A) Results for L.E.L. are on the left. (B) Results for J.M. are on the right.

adapting field sizes. These results are presented in Figures 2 and 3, respectively. Both figures reveal the same kind of trends found in Figure 1. Specifically, both incremental threshold and the drop in threshold during early dark adaptation appear to be the greatest when the test and adapting fields are similar in size. Also, no further decreases in the magnitude of either kind of threshold are found for adapting fields that differ from the test size by a factor of two.

A comparison of the results of L.E.L. indicates that the magnitude of the threshold drop, as well as the threshold elevation, depends upon the size of the test field. When the borders of the test and adapting fields are coincident, threshold elevation is greater for the smallest stimuli (15.8 $\mathrm{min}$ ) than for the larger stimuli.

\section{DISCUSSION}

The results of the present experiment indicate that background size influences the magnitude of the incremental threshold as well as the magnitude of the temporal course of early dark adaptation. It is quite apparent that the present results cannot be accounted for in terms of local light or single photoreceptors. In all experimental conditions, adapting fields equal to and larger than the test field adapted the photoreceptors responsive to the test spot to the same extent. Consequently, removing the adapting field should have produced similar time courses of early dark adaptation for a particular size of test field, regardless of the adapting field size. This, however, was not the case.

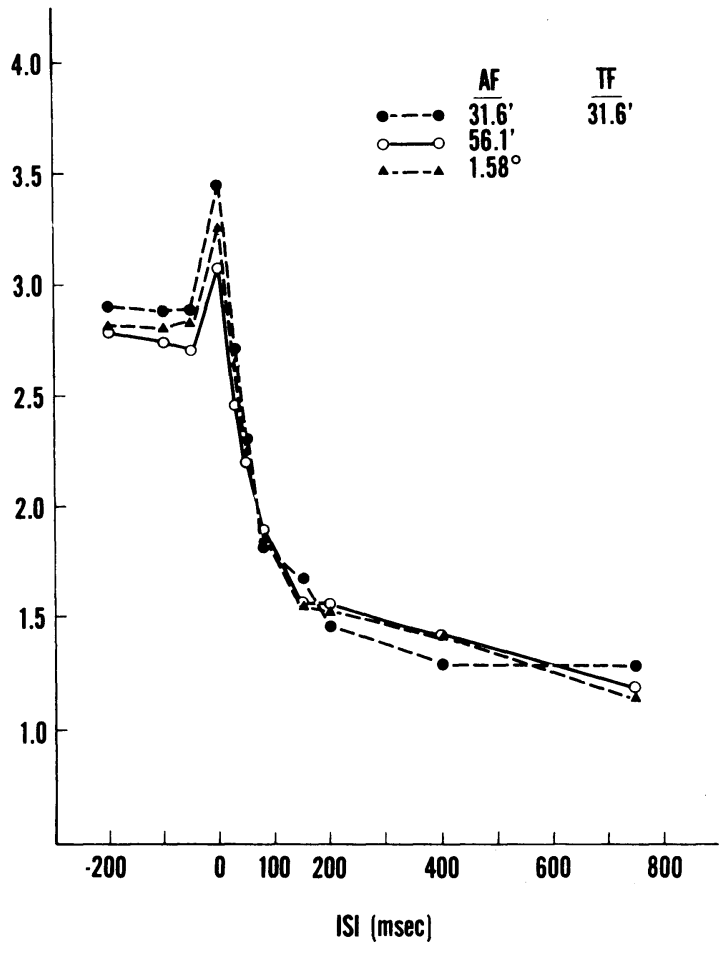

Figure 2. Log threshold as a function of ISI for a $31.6-\mathrm{min}$ test field. Results for L.E.L. 


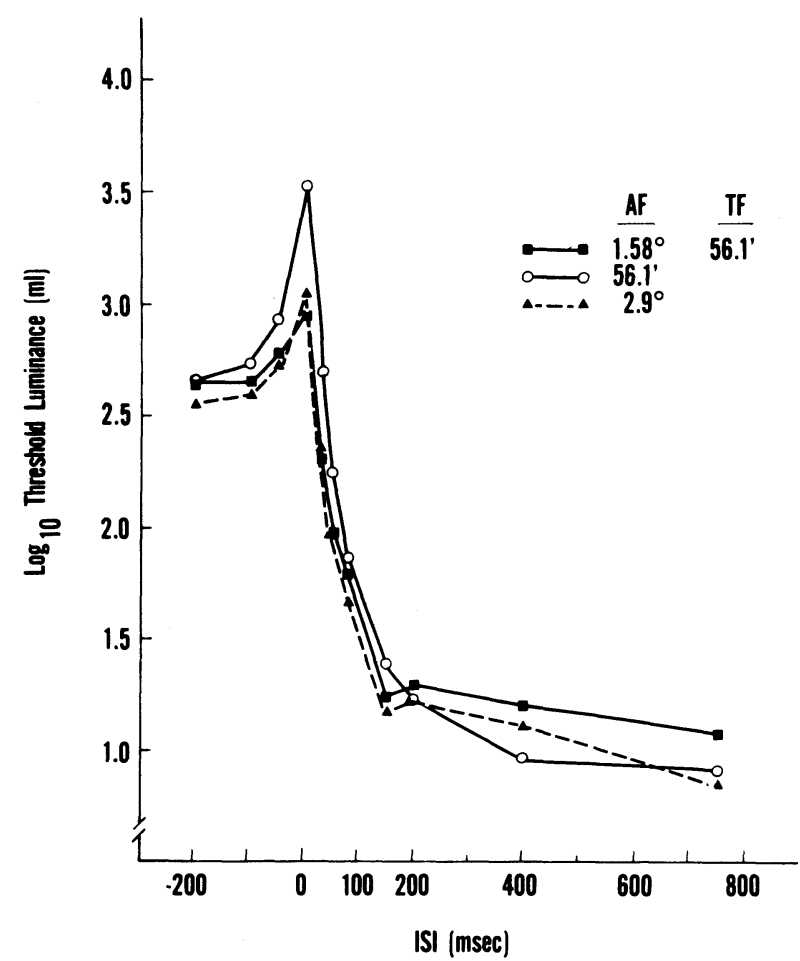

Figure 3. Log threshold as a function of ISI for a 56.1-min test field. Results for L.E.L.

Adapting fields similar in size to the test field produced greater threshold elevations and longer time courses of early dark adaptation than did adapting fields that were larger than the test field. A comparison of the results for conditions in which adapting fields and test fields were similar in relative size but different in absolute size indicates that the absolute magnitude of the stimulus configuration also influences both kinds of threshold measures (see also Kitterle \& Leguire, 1975). Smaller configurations yield larger incremental thresholds and longer time courses than do larger configurations. Thus, border proximity alone does not determine the magnitude of threshold elevation and drop. That is, early dark adaptation does not solely reflect a recovery from lateral border inhibition. It appears that the way light is spatially integrated rapidly changes over this time course, which seems to also indicate a progressive change in the functional organization of the visual system influences threshold during early dark adaptation.

\section{REFERENCES}

Frumkes, T. E., \& Kraft, L. L. Spatial interaction with different diameter stimuli matched on the basis of threshold, luminance, or total flux. Journal of the Optical Society of America, 1972, 62, 1117-1118.

Frumkes, T. E., \& Sturr, J. F. Spatial and luminance factors determining visual excitability. Journal of the Optical Society of America, 1968, 58, 1657-1662.

Kitterle, F. L., \& Leguire, L. E. The effect of borders and contours on threshold during early dark adaptation. Vision Research, 1975, 15, 1217-1224.

Markoff, J. I., \& Sturr, J. F. Spatial and luminance determinants of the incremental threshold under monoptic and dichoptic viewing. Journal of the Optical Society of America, 1971, 61, 1530-1537.

Ratoosh, P., \& Graham, C. H. Areal effects in foveal brightness discrimination. Journal of Experimental Psychology, 1951, 42, 367-375.

(Received for publication October 24, 1980.) 